\title{
EBV-LMP1 promotes radioresistance by inducing protective autophagy through BNIP3 in nasopharyngeal carcinoma
}

\author{
San Xu ${ }^{1,2,3}$, Zhuan Zhou ${ }^{1,2}$, Xingzhi Peng ${ }^{1,2}$, Xuxiu Tao ${ }^{1,2}$, Peijun Zhou ${ }^{1,2}$, Kun Zhang ${ }^{1}$, Jinwu Peng ${ }^{4}$, Dan Li ${ }^{5}$, \\ Liangfang Shen ${ }^{1}$ and Lifang Yang $\mathbb{B}^{1,2,6}$
}

\begin{abstract}
Studies have indicated that dysfunction of autophagy is involved in the initiation and progression of multiple tumors and their chemoradiotherapy. Epstein-Barr virus (EBV) is a lymphotropic human gamma herpes virus that has been implicated in the pathogenesis of nasopharyngeal carcinoma (NPC). EBV encoded latent membrane protein1 (LMP1) exhibits the properties of a classical oncoprotein. In previous studies, we experimentally demonstrated that LMP1 could increase the radioresistance of NPC. However, how LMP1 contributes to the radioresistance in NPC is still not clear. In the present study, we found that LMP1 could enhance autophagy by upregulating the expression of BCL2/ adenovirus E1B $19 \mathrm{kDa}$ protein-interacting protein 3 (BNIP3). Knockdown of BNIP3 could increase the apoptosis and decrease the radioresistance mediated by protective autophagy in LMP1-positive NPC cells. The data showed that increased BNIP3 expression is mediated by LMP1 through the ERK/HIF1a signaling axis, and LMP1 promotes the binding of BNIP3 to Beclin1 and competitively reduces the binding of $\mathrm{BCl}-2$ to Beclin1, thus upregulating autophagy. Furthermore, knockdown of BNIP3 can reduce the radioresistance promoted by protective autophagy in vivo. These data clearly indicated that, through BNIP3, LMP1 induced autophagy, which has a crucial role in the protection of LMP1-positive NPC cells against irradiation. It provides a new basis and potential target for elucidating LMP1-mediated radioresistance.
\end{abstract}

\section{Introduction}

Nasopharyngeal carcinoma (NPC) is the most malignant tumor in the Southeast Asia region and south China. The primary clinical treatment of NPC is radiotherapy. However, radioresistance and metastasis affect the treatment outcomes and remain the key problems related to survival for NPC patients ${ }^{1,2}$. Epstein-Barr virus (EBV) is a lymphotropic human gamma herpesvirus which has been

\footnotetext{
Correspondence: Lifang Yang (yanglifang@csu.edu.cn)

${ }^{1}$ Department of Oncology, Key Laboratory of Carcinogenesis and Cancer Invasion of Ministry of Education, Xiangya Hospital, Central South University, Changsha, China

${ }^{2}$ Cancer Research Institute, School of Basic Medicine Science, Central South University, Changsha, China

Full list of author information is available at the end of the article

These authors contributed equally: San Xu, Zhuan Zhou

Edited by G. M. Fimia
}

implicated in the pathogenesis of several human malignancies, including Burkitt's and Hodgkin's lymphomas, gastric carcinoma, and NPC ${ }^{3}$. EBV encoded latent membrane protein 1 (LMP1) is thought to play a key role in the pathogenesis of NPC. It triggers several important signal transduction pathways, such as NF-kB, MAPK, JAK/ STAT, PI3K/AKT, and others involved in the proliferation, apoptosis, and metastasis of tumor cells ${ }^{4,5}$. In previous studies, we experimentally demonstrated that LMP1 has an important role in the regulation of the radioresistance of NPC cells ${ }^{6-8}$. However, the mechanism of LMP1-mediated radioresistance is not entirely clear.

Autophagy is a conserved biological process for digestion and recycling of cytoplasmic constituents in eukaryotic cells, which is essential for maintaining genomic integrity and homeostasis ${ }^{9}$, and can be induced in

\section{(c) The Author(s) 2021}

(c) (i) Open Access This article is licensed under a Creative Commons Attribution 4.0 International License, which permits use, sharing, adaptation, distribution and reproduction c. in any medium or format, as long as you give appropriate credit to the original author(s) and the source, provide a link to the Creative Commons license, and indicate if changes were made. The images or other third party material in this article are included in the article's Creative Commons license, unless indicated otherwise in a credit line to the material. If material is not included in the article's Creative Commons license and your intended use is not permitted by statutory regulation or exceeds the permitted use, you will need to obtain permission directly from the copyright holder. To view a copy of this license, visit http://creativecommons.org/licenses/by/4.0/. 


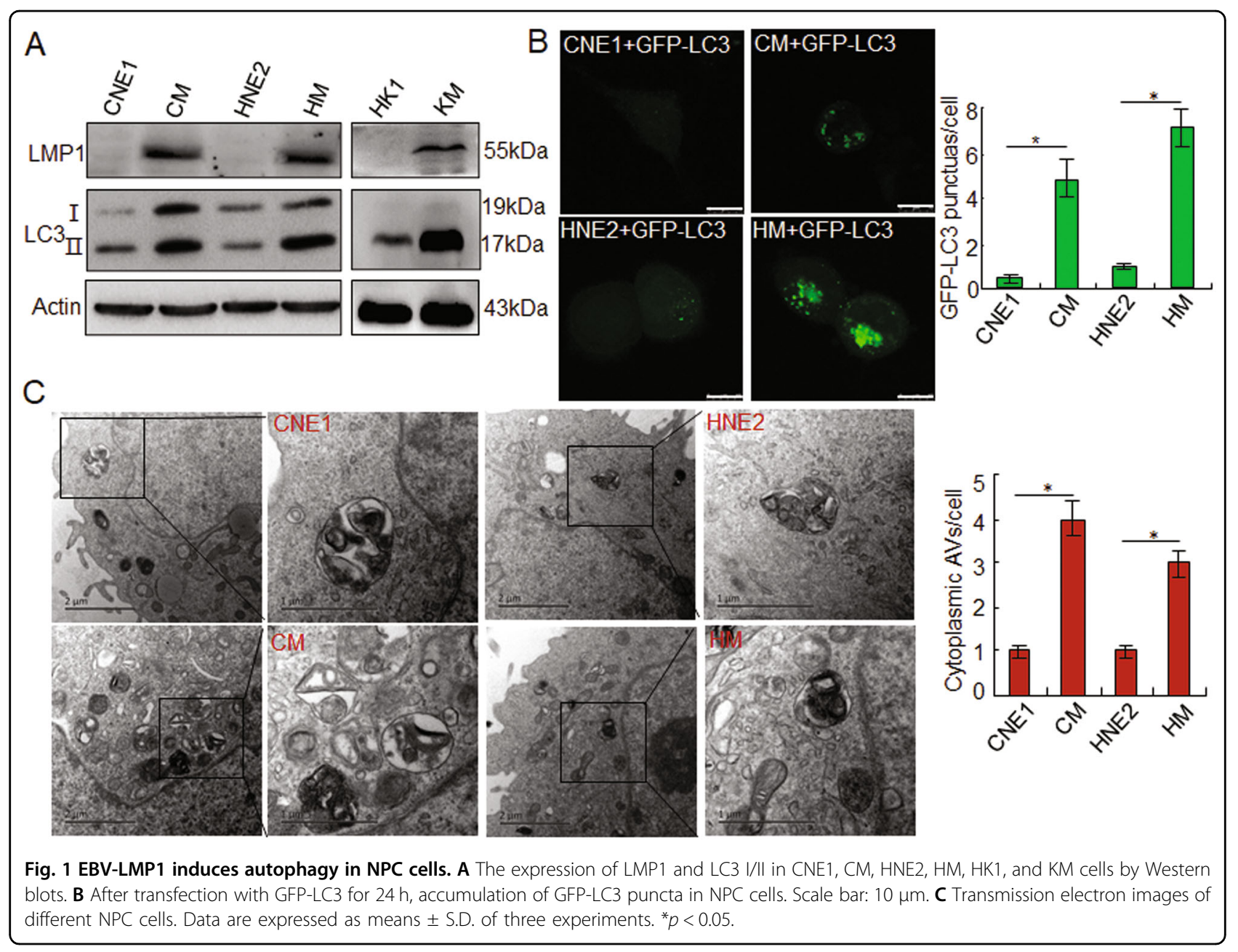

response to various conditions including nutrient deprivation, metabolic stress, and hypoxia to adapt cellular conditions for survival ${ }^{10}$. Accumulating evidence has indicated that autophagy is important in the regulation of cancer development and progression, and in determining the response of tumor cells to anticancer therapies in a wide variety of tumors, including lung cancer, breast cancer, malignant glioma, and pancreatic cancer ${ }^{11,12}$. For example, in pancreatic cancer cells, defective SMAD4 is responsible for radioresistance through increased levels of radiation-induced autophagy ${ }^{13}$. The studies by Chen $\mathrm{X}$ et al. show that hypoxia enhanced cell radioresistance by increasing the induction of autophagy in non-small cell lung cancer ${ }^{14}$. These studies suggest that autophagy may play an important role in tumor radioresistance, although its mechanism remains unclear.

In the B lymphocytes, LMP1 induces autophagy in a dose-dependent manner and, thus, the inhibition of autophagy in EBV-positive cells leads to a decreased ability to form colonies ${ }^{15}$. Therefore, whether LMP1 is involved in the regulation of autophagy of NPC, and how
LMP1-mediated autophagy plays a role in tumor radioresistance, needs further exploration. In this study, we found that LMP1 could enhance the autophagy of NPC cells, and the BCL2/adenovirus E1B $19 \mathrm{kDa}$ proteininteracting protein 3 (BNIP3) was identified as a key modulator of LMP1-induced autophagy. Furthermore, the ERK/HIF1 $\alpha$ signaling axis was confirmed to be crucial for LMP1-induced upregulation of BNIP3, and regulation of autophagy by LMP1-induced BNIP3 is in a Beclin1dependent manner. These findings provide a potential strategy for the sensibilization therapy of LMP1-positive NPC, by using the inhibitor of autophagy or BNIP3.

\section{Results}

EBV-LMP1 induces autophagy in NPC cells

To investigate whether EBV-LMP1 induces autophagy in NPC cells, we used LMP1-positive cells, CM, HM and KM, and LMP1-negative cells, CNE1, HNE2, and HK1, for analysis. The data indicated that in the LMP1-positive cells, the LC3-II level was significantly increased compared to the LMP1-negative cells (Fig. 1A). Further, 
immunofluorescence experiments also found that transfection with GFP-LC3 plasmids resulted in a significant increase of LC3 puncta in CM and HM cells compared to CNE1 and HNE2 cells (Fig. 1B). Moreover, to further investigate the effects of LMP1 on autophagy, transmission electron microscopy was utilized. A greater number of double-membrane vesicles accumulated in the CM and HM cells compared to the control group (Fig. 1C). These results suggested that LMP1 could induce autophagy in NPC cells.

\section{LMP1 induces autophagy through BNIP3}

To determine which molecules are involved in enhancing LMP-mediated autophagy in NPC cells, PCR-Array screening was applied to investigate the alteration of autophagy associated genes at the mRNA level in LMP1positve CM cells in comparison with LMP1-negative CNE1 cells. The results showed that, among the 88 genes, the transcription level of BNIP3 was most obviously increased in the CM and HM cells compared to the CNE1 and HNE2 cells (Fig. 2A). To further confirm the upregulation of BNIP3 in LMP1-positive cells, the mRNA and protein level of LMP1 and BNIP3 were detected in the NPC cells. The data showed that BNIP3 expression in LMP1 positive NPC cells was significantly higher than in LMP1 negative cells at both the mRNA $(p<0.05)$ and protein level (Fig. 2B, C). After transient transfection with pGV141LMP1-wt plasmids in CNE1,HNE2, and HK1 cells, the results indicated that LMP1 could induce BNIP3 and LC3II expression in NPC cells, suggesting LMP1 could induce autophagy related to BNIP3 (Fig. 2D, E).

In order to determine whether the BNIP3 involved in LMP1 mediated autophagy, targeting BNIP3 shRNA were transfected into CM, HM, and KM cells. The data showed that knockdown of BNIP3 could significantly reduce the LMP1 mediated LC3II expression (Figs. 3A and S1). Immunofluorescence experiment results also indicated that knockdown of BNIP3 could obviously suppress the LC3 puncta, which was induced by the EBV-LMP1 (Fig. 3B). Furthermore, IHC staining of 27 clinical patient samples indicated the expression of BNIP3 and LC3 II in LMP1 positive tissues (Fig. 3C). Spearman correlation analysis between LMP1, BNIP3, and LC3 II was confirmed based on the IHC staining score. The results showed that LMP1 and BNIP3 $(r=0.5539, p=$ 0.0027), LMP1 and LC3 II $(r=0.0 .3920, p=0.0431)$, and BNIP3 and LC3 II ( $r=0.5228, p=0.0051)$ had a positive correlation. These findings suggested that LMP1 induces autophagy through BNIP3 in NPC cells.

\section{LMP1 increases radioresistance of NPC cells through BNIP3}

The function of BNIP3 has been associated with both cell death and cell survival ${ }^{16}$. To determine the influence of BNIP3 mediated by LMP1 on NPC cell proliferation, the MTS results showed that the proliferation ability of LMP1-positive NPC cells was stronger than that of LMP1-negative NPC cells, but the proliferation ability of $\mathrm{CM}$ and HM cells with knockdown of BNIP3 showed no

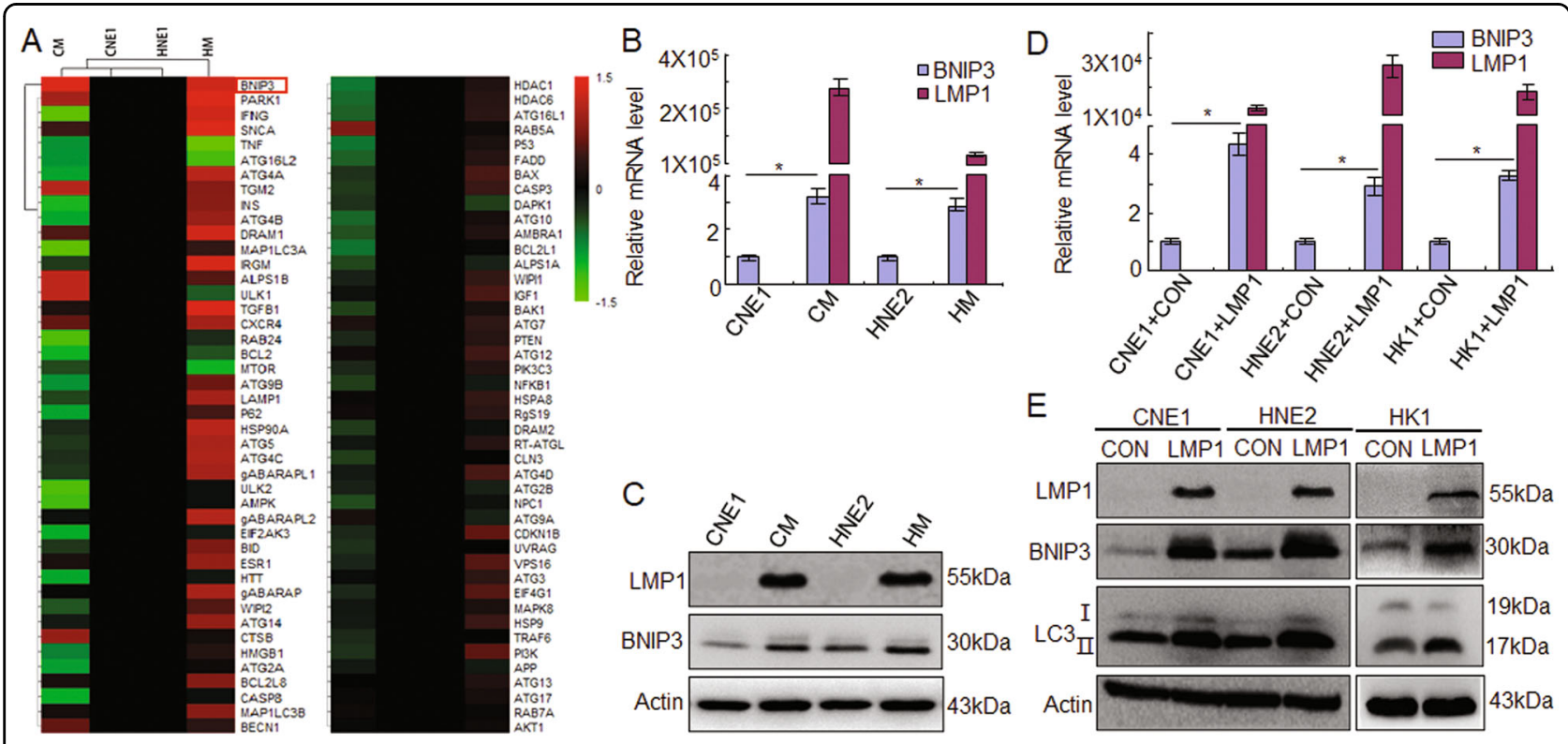

Fig. 2 LMP1 increases BNIP3 expression in NPC cells. A A PCR array was used to detect changes in the expression of autophagy-related genes in LMP1-positive NPC cells compared with LMP1-negative NPC cells. B Quantitative RT-PCR and C Western blots were performed to analyze LMP1 and BNIP3 expression in NPC cells. D Quantitative RT-PCR and $\mathbf{E}$ Western blots were performed to analyze LMP1, BNIP3, and LC3 expression after transfection with LMP1 in NPC cells. CON: negative control, Data are expressed as means \pm S.D. of three experiments. ${ }^{*} p<0.05$. 


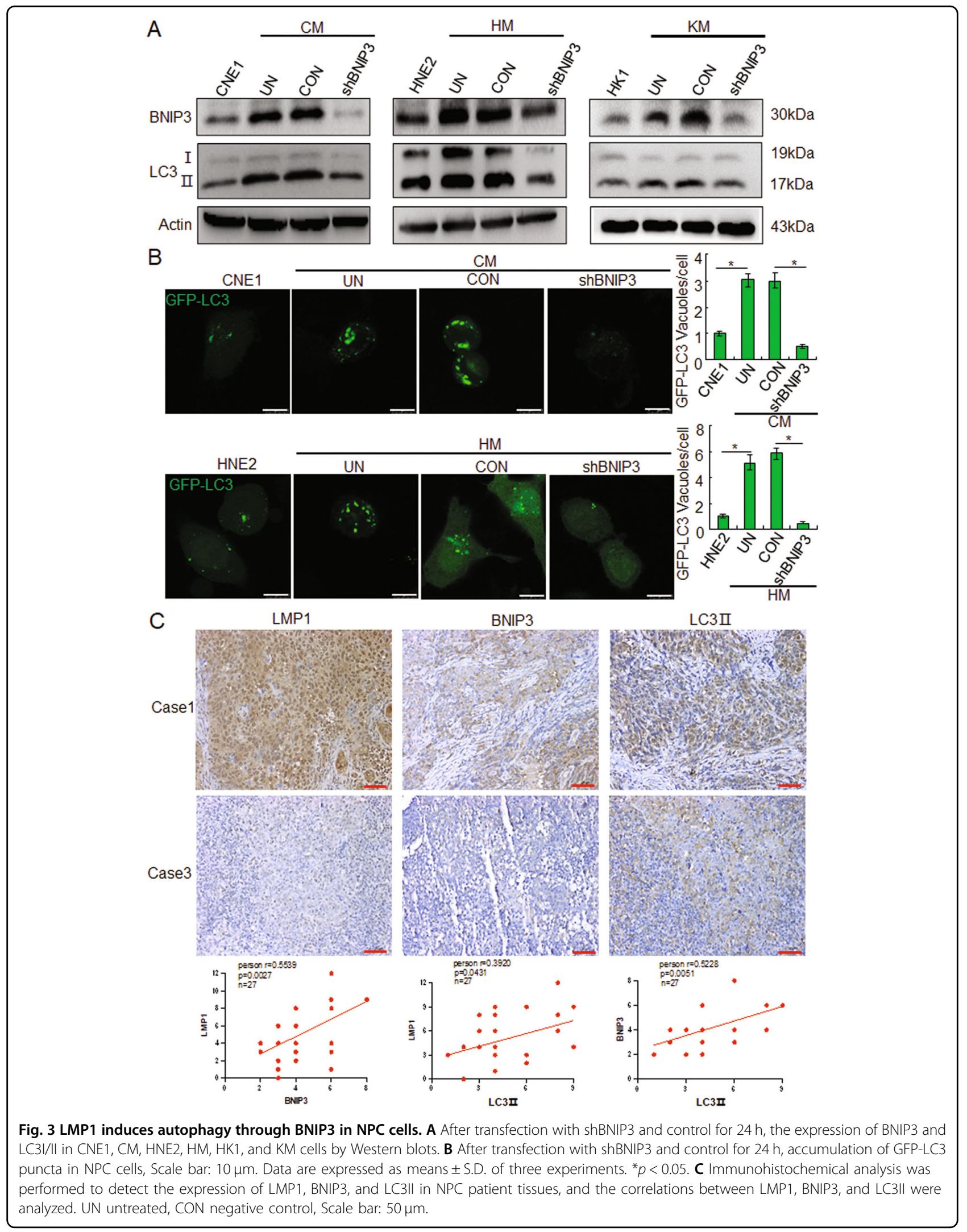




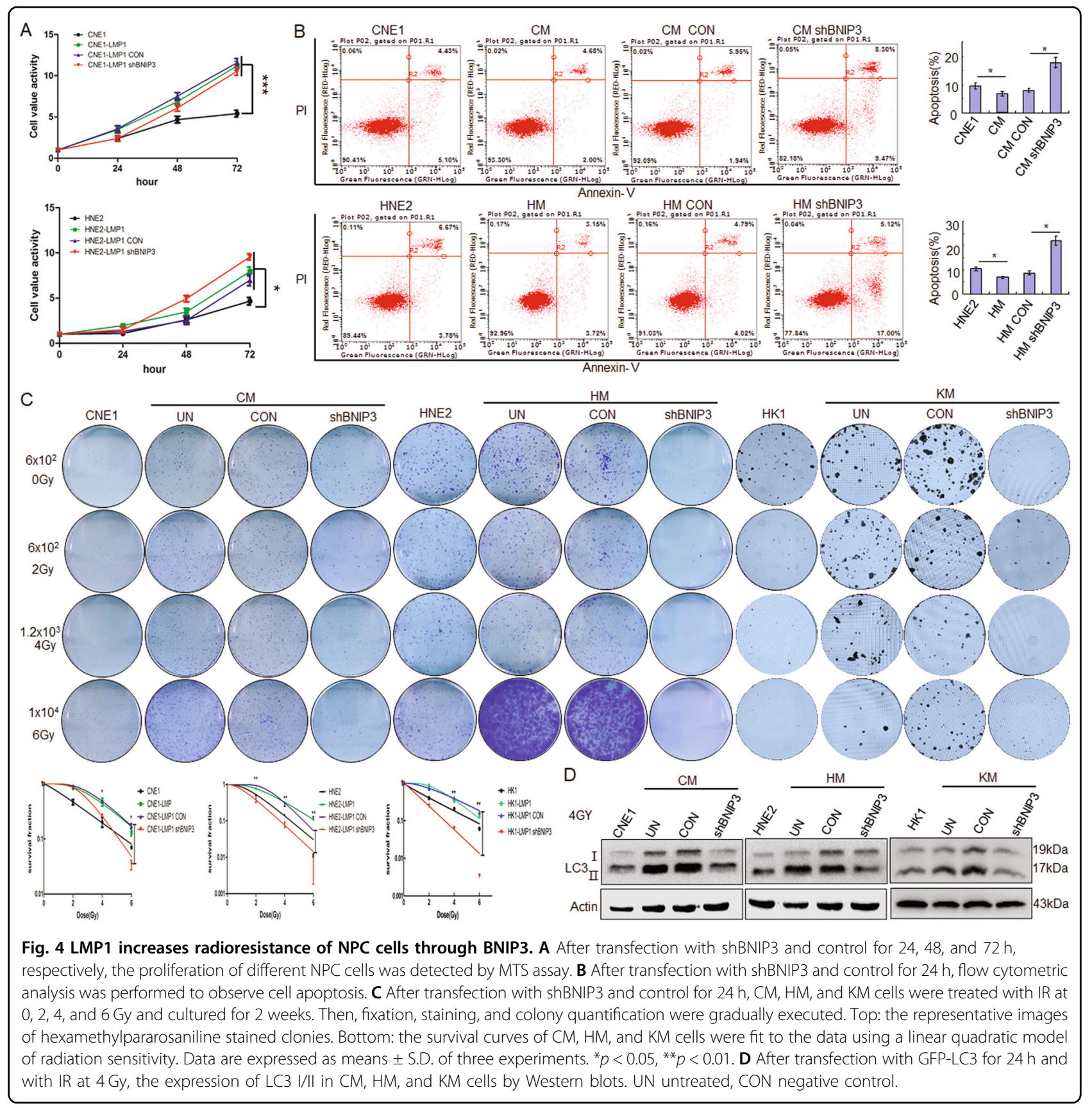

obvious change compared to the control group (Fig. 4A). Further, the flow cytometry results showed that the apoptosis of LMP1-positive NPC cells was lower than that of LMP1-negative NPC cells, and there was increased apoptosis of CM and HM after knockdown of BNIP3 compared to the control group $(p<0.05)$ (Fig. 4B). These results show that LMP1-induced BNIP3 can inhibit apoptosis and have no obvious effect of proliferation in NPC cells. Clinically, the radioresistance of NPC is the key factor affecting the therapeutic effect ${ }^{17}$, and autophagy is an important mechanism to regulate radioresistance ${ }^{12}$.
Therefore, we want to know whether BNIP3 and its induced autophagy are involved in the regulation of radioresistance. The colony formation assays results showed that colony formation of LMP1-negative NPC cells, whether treated or not treated with irradiation, was weaker than that of LMP1-positive NPC cells $(p<0.01)$, which indicated that LMP1 could increase cellular radioresistance. After knockdown of BNIP3, colony formation of LMP1-positive CM, HM, and KM cells were significantly decreased compared to control groups $(p<0.01)$ (Fig. 4C). Meanwhile, Western blot results also 
showed that knockdown of BNIP3 could significantly inhibit LC3II expression (Fig. 4D). These results suggest that BNIP3 could increase the radioresistance through increased autophagy in NPCs.

EBV-LMP1 induces BNIP3 through the ERK/HIF1a pathway

Some studies have reported that LMP1 triggers several signal pathways, such as MAPK, JAK/STAT, PI3K/AKT, $\mathrm{NF}-\mathrm{KB}$, and others, to regulate multiple functions in NPC cells $^{4,5}$. To determine which pathway is mainly responsible for inducing BNIP3 expression and autophagy, the inhibitors targeting PI3K/AKT (LY294002), JAK3/STAT (WHIP131), p38 (SB203580), ERK (PD98059), and JNKs (SP600125) were used. The results showed that in CM cells, BNIP3 expression decreased after treatment with LY294002 and PD98059. Importantly, LC3II expression decreased only after treatment with PD98059. In HM cells, the BNIP3 expression decreased after treatment with LY294002, WHIP131, and PD98059, and the LC3II expression also decreased only after treatment with PD98059 (Figs. 5A and S2). These results indicate that the ERK signaling pathway is mainly responsible for BNIP3 upregulation and autophagy induced by LMP1 in NPC cells. Furthermore, HIF1 $\alpha$ is a key molecule for regulating BNIP $^{18-20}$. Therefore, we analyzed whether HIF $1 \alpha$ is involved in regulation of LMP1 on BNIP3in LMP1positive NPC cells with HIF1 $\alpha$ inhibitor 2ME2 treatment, and the data showed that with the inhibition of HIF1 $\alpha$, BNIP3 mRNA and protein expression decreased accordingly (Fig. 5B, C). Moreover, the results indicated that HIF1 $\alpha$ decreased, but the p-ERK expression did not change after 2ME2 treatment, while both the p-ERK and HIF1 $\alpha$ decreased after PD98059 treatment in CM, HM and KM cells (Fig. 5D). These results suggest the ERKHIF1 $\alpha$ signal axis is responsible for LMP1 induced BNIP3 expression and autophagy in NPC cells.

\section{LMP1-induced BNIP3 triggers autophagy through a dissociated Bcl-2-Beclin1 complex}

It has been proven that the role of BNIP3 in autophagy has three main forms: the first is that under the condition of hypoxia, BNIP3 forms dimer, which locates on the outer membrane of the mitochondria and causes mitochondrial damage, inducing autophagic cell death. The second is that BNIP3 dimer locates on the mitochondria and interacts with the autophagosome-localized LC3, and causes mitophagy. The third is that BNIP3 can destroy the structure of Bcl-2 and Beclin1 complex and release Beclin1 through the competitive binding with Bcl-2, thus promoting autophagy ${ }^{21}$. To clarify which mechanism is involved in LMP1-induced protective autophagy, the location of BNIP3 and the relationship among BNIP3, Beclin1, LC3, Bcl-2, and autophagy were investigated. It was found that BNIP3 was located on the mitochondrial membrane and LMP1 promoted BNIP3 expression in NPC cells by immunofluorescence analysis in CM and KM cells (Fig. 6A). Further, the immunoprecipitation analysis showed that LMP1 increased the Beclin1, but not LC3, to bind with BNIP3 (Fig. 6B). Furthermore, we found that LMP1 dramatically decreased the binding between Bcl-2 and Beclin1 compared to the control, through immunoprecipitation. However, only part of the Beclin1 was displaced with BNIP3, so the Beclin1-Bcl-2 complex was still detectable at a reduced level in the LMP1positive NPC cells (Fig. 6C). Taken together, these results suggest that LMP1-induced BNIP3 expression triggers protective autophagy through increasing the disruption of the Bcl-2-Beclin1 complex.

\section{LMP1-induced BNIP3 increases radioresistance of NPC in vivo}

To investigate whether LMP1-induced BNIP3 increases radioresistance through protective autophagy in vivo, animal experiments were performed. As shown in Fig. 7A-C, although knockdown of BNIP3 alone or radiation treatment could inhibit tumor growth compared with control groups $(p<0.05)$, the combined effect of BNIP3 and radiation significantly reduced the tumor growth $(p<$ 0.001). Further, IHC staining of the tumor xenograft tissue showed that knockdown of BNIP3 could effectively reduce the expression of LC3II, although radiation could promote the expression of LC3 to a certain extent (Fig. 7D). These data demonstrated that LMP1-induced BNIP3 could increase the radioresistance through protective autophagy in vivo.

We thus propose a simple model (Fig. 7E) in which the LMP1-induced BNIP3 through ERK/HIF1 $\alpha$ are crucial to the disruption of an interaction between $\mathrm{Bcl}-2$ and Beclin1, thereby releasing the negative autophagic blockade, and further triggering the radioresistance of the NPC tumor.

\section{Discussion}

Autophagy has been described as a form of cell death for a variety of cell types. However, in the context of hypoxia, nutrient depletion, or growth factor deprivation, it is clear that autophagy is crucial in maintaining cellular ATP production and macromolecular synthesis and, therefore, represents an essential pro-survival pathway ${ }^{10}$. Therefore, there are two opposing views about the relationship between autophagy and radiotherapy resistance. Some researchers think that autophagy can promote radiosensitivity, but others disagree. For example, Kuwahara et al. reported that an autophagy inhibitor, 3-methyladenine, induced radioresistance of liver cancer cells ${ }^{22}$. Liang's group found that induction of autophagy with rapamycin promoted glioma-initiating cell sensitivity to irradiation ${ }^{23}$. Conversely, Chaachouay et al. found that 


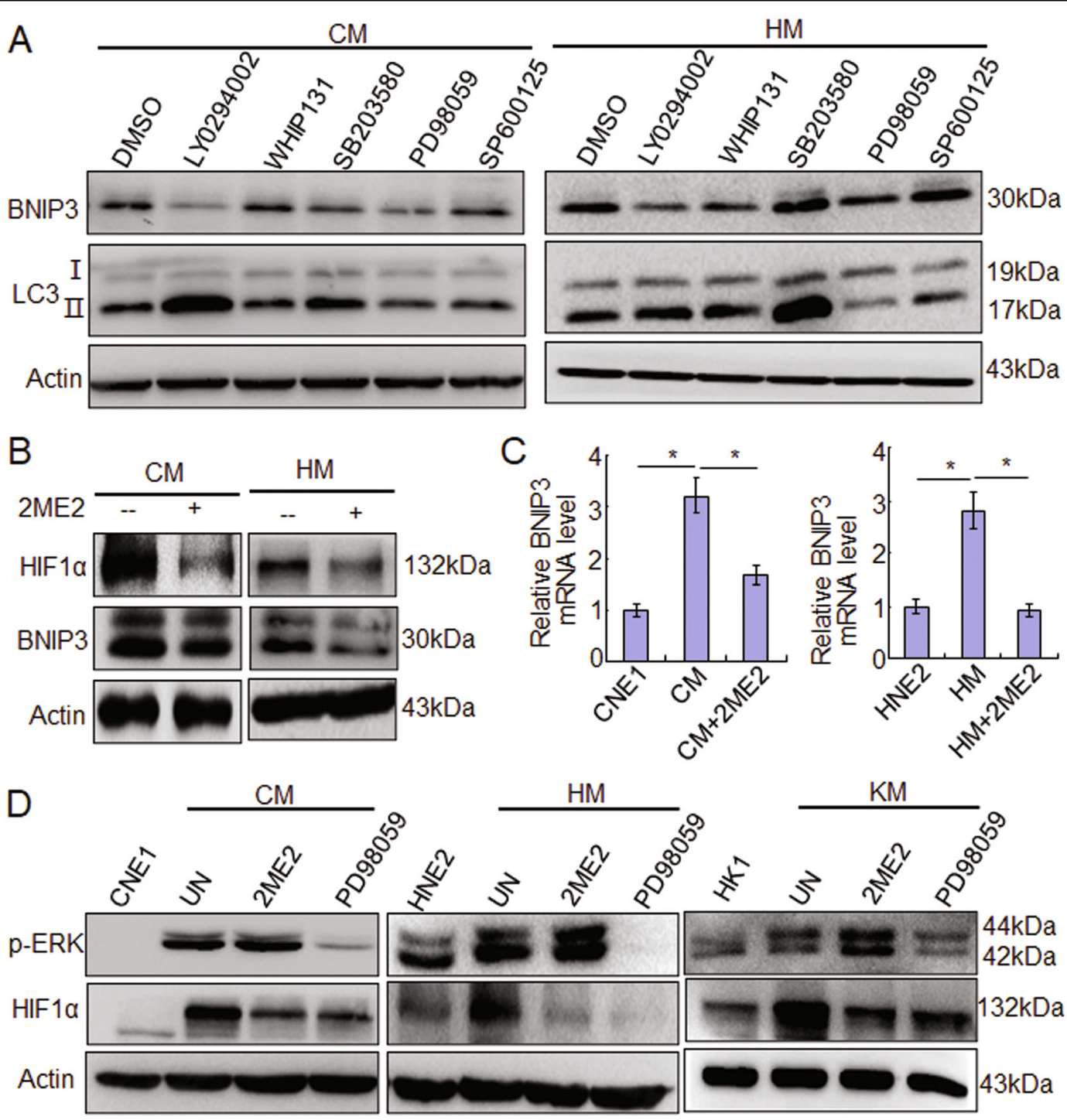

Fig. 5 EBV-LMP1 induces BNIP3 through the ERK/HIF1a pathway. A After treatment of different inhibitors for $24 \mathrm{~h}$, the expression of BNIP3 and LC3I/II in NPC cells by Western blots. B After treatment of 2ME2 for $24 \mathrm{~h}$, the expression of HIF1a and BNIP3 in NPC cells by Western blots. C After treatment of $2 \mathrm{ME} 2$ for $24 \mathrm{~h}$, the mRNA expression of BNIP3 in NPC cells by quantitative RT-PCR. Data are expressed as means \pm S.D. of three experiments. ${ }^{*} p<0.05$. D After treatment of 2 ME2 or PD 98059 for $24 \mathrm{~h}$, the expression of p-ERK and HIF1a in CM, HM, and KM cells by Western blots. UN untreated.

autophagy contributes to resistance of breast cancer cells to ionizing radiation ${ }^{24}$. Ko et al. also found that autophagy can inhibit radiosensitization in vitro, yet reduces radioresponses in vivo due to deficient immunogenic signaling $^{25}$. In our present study, the results showed that EBV-LMP1 was involved in the radioresistance of NPC cells by promoting cell protective autophagy.

The BH3-only family member BNIP3 has been described as pro-death, as well as having pro-autophagic and protective functions, depending on the type of stress and cellular context ${ }^{16}$. Most studies have reported that BNIP3 can induce apoptosis. For example, Verticillin A induced
BNIP3 expression in human colon carcinoma, and hepatoma has been reported to correlate with apoptosis ${ }^{26}$. Conversely, there are still some studies suggesting that BNIP3 can inhibit the apoptosis of tumor cells ${ }^{27,28}$. On the other hand, BNIP3 has been reported to induce autophagy in tumor cells. However, whether this leads to cell death or survival is controversial, as the induction of autophagy by BNIP3 is associated with autophagic cell death, but it has been found to have a protective effect in some conditions. Chourasia et al. reported that mitophagy defects arising from BNIP3 loss promote mammary tumor progression to metastasis ${ }^{29}$. Wu et al. found that BNIP3 

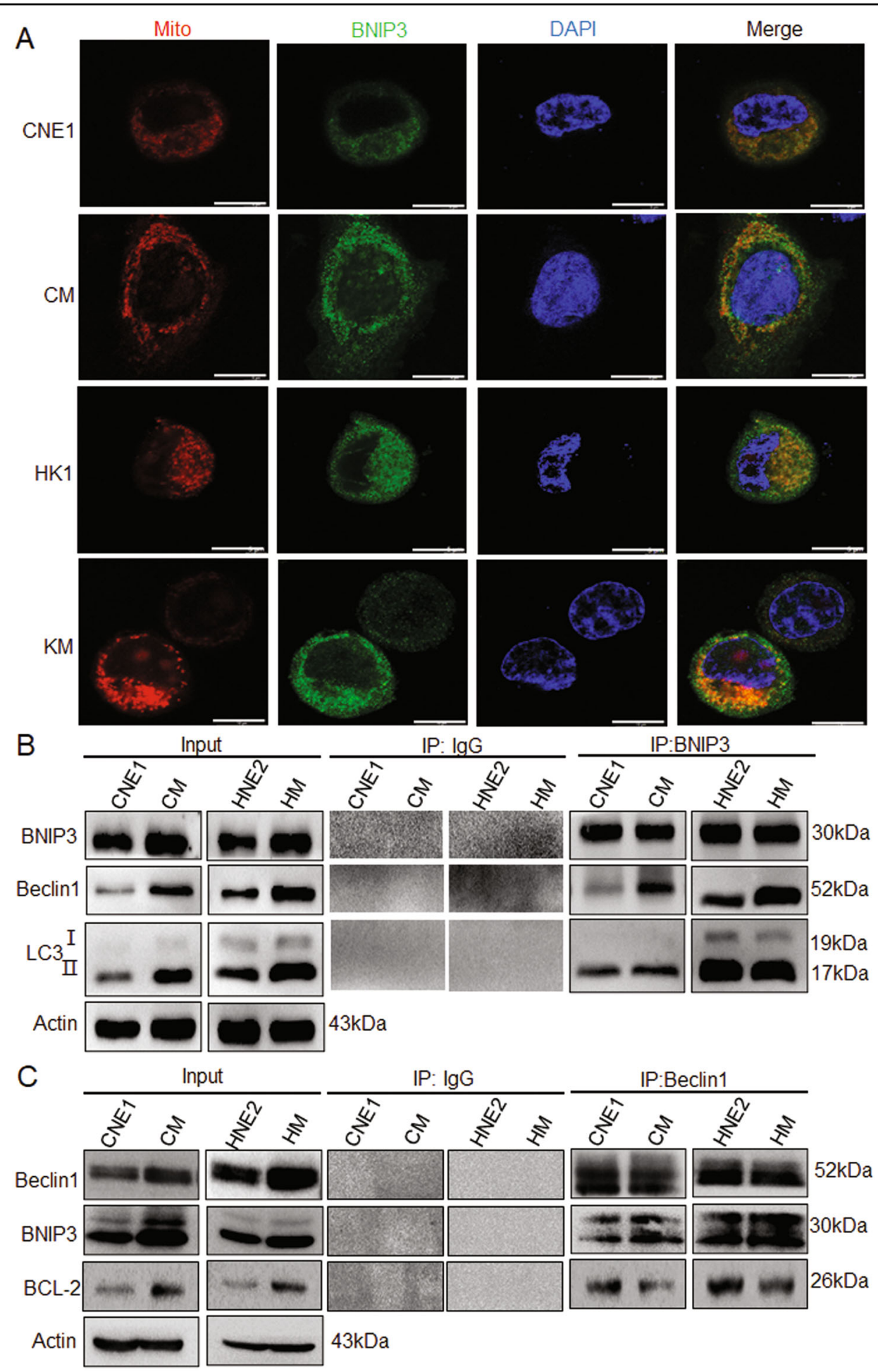

Fig. 6 LMP1-induced BNIP3 triggers autophagy through a dissociated Bcl-2-Beclin1 complex. A After MitoTracker Red staining, the colocalization of BNIP3 in NPC cells CNE1, CM, HK1, and KM was detected by immunofluorescence assay. Scale bar: $10 \mu \mathrm{m}$. B The interaction of BNIP3 with Beclin1 and LC3II was detected by Co-IP. C The interaction of Beclin1 with BNIP3 and Bcl-2 was detected by Co-IP. 

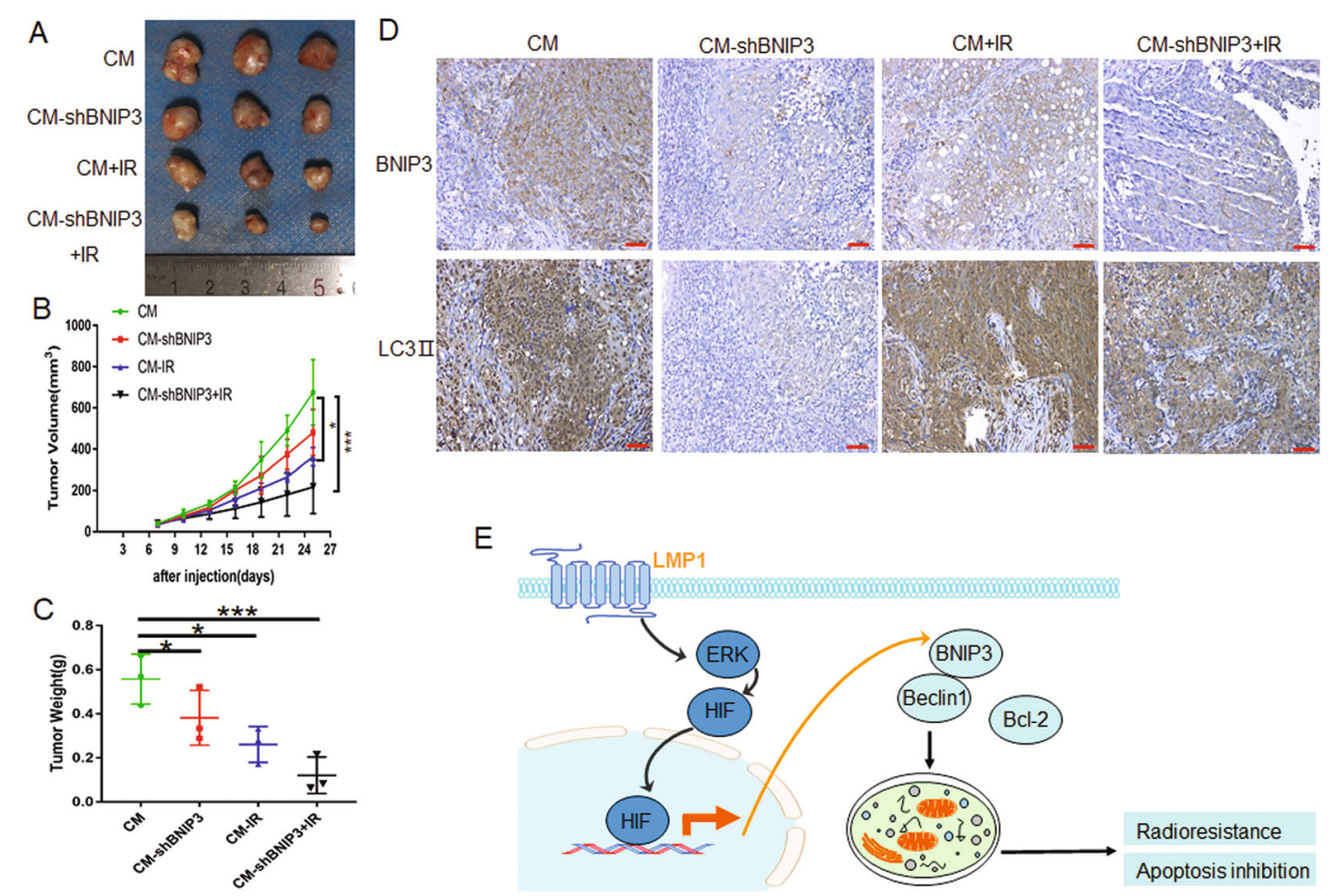

Fig. 7 LMP1-induced BNIP3 increases radioresistance of NPC in vivo. The xenograft model was established using CM cells or CM-shBNIP3 cells. When the tumor volume reached $60-100 \mathrm{~mm}^{3}, 6 \mathrm{~Gy}$ local irradiation was administered. A At the experimental end point, tumor xenografts were dissected and photographed. B Tumor volume was measured every 3 days after injection. $\mathbf{C}$ Tumor weight was measured at the experimental end point. ${ }^{*} p<0.05,{ }^{* * *} p<0.001$. D Representative immunohistochemistry (IHC) staining of BNIP3 and LC3II expression in CM xenografts in the groups indicated in A. Scale bar: $50 \mu \mathrm{m}$. E A schematic illustration of the LMP1 promoting radioresistance by inducing autophagy through ERK/HIF1a/BNIP3 in NPC.

can inhibit the growth of tumor cells by regulating autophagy in the process of Ras-regulated tumor growth $^{30}$. In contrast, Nollet et al. found that the integrins $\alpha 6 \beta 1$ and Bnip3 promote survival of CRPC cells selectively on laminin, through the induction of autophagy and mitophagy $^{31}$. Li et al. also found that p53 repressed BNIP3 transcription and activity, leading to mitophagy arrest. The defective mitophagy impaired mitochondrial homeostasis, evoked cellular oxidative stress, and initiated mitochondrial apoptosis ${ }^{32}$. These studies fully illustrate the diversity of action modes and biological functions of BNIP3 under different conditions.

In our present study, although knockdown of BNIP3 had no significant effect on NPC cell survival, the significant enhancement of radiosensitivity following BNIP3 knockdown indicates that BNIP3-induced autophagy is indispensable for cell survival under IR treatment. In addition to autophagy, BNIP3 inhibition also leads to the appearance of apoptosis. It may be that defective mitophagy impairs mitochondrial homeostasis, therefore initiating apoptosis. For NPC, LMP1 mediated BNIP3induced autophagy appears to be a protective process, probably acting as a warning signal for cells to anticipate irradiation stress.
At present, many signaling pathways have been demonstrated as being involved in regulating BNIP3. Sun et al. found that the activation of the ERK/HIF-1 signaling pathway in liver cancer cells can promote BNIP3 dependent autophagy, and participate in cell apoptosis resistance $^{19}$. Riis et al. confirmed that IGF-1 induced BNIP3 expression through AKT1-mediated inhibitory phosphorylation of GSK-3 $\beta^{33}$. We also observed that the increased autophagy in the LMP1-positive cells is mainly mediated through the extracellular ERK/HIF1 $\alpha$ signaling axis. It has been shown that there are several ways BNIP3 mediates autophagy; the first is that under hypoxia, BNIP3 causes mitochondrial injury induced autophagic cell death $^{21}$. The second is that BNIP3 dimers locate the mitochondria and interact with the autophagosomelocalized LC3, causing mitophagy ${ }^{34,35}$. The third is that BNIP3 can destroy the structure of Bcl-2 and Beclin1 complexes and release Beclin1, thus promoting autophagy $^{21,31}$. In this report, we demonstrated that LMP1induced autophagy is controlled by BNIP3. In particular, BNIP3 triggers autophagy through dissociated Bcl-2Beclin1 complexes, and this protective autophagy might be required for the protection of NPC cells from irradiation-induced cell death. 
In previous studies, we demonstrated that LMP1 plays an important role in the radioresistance of NPC cells. LMP1 represses the DNA damage response (DDR) through DNA-dependent protein kinase DNA-PK/ AMPK signaling, and then promotes resistance to apoptosis induced by irradiation ${ }^{6}$. Meanwhile, LMP1 promotes hexokinase 2 (HK2) through c-Myc, and the upregulation of $\mathrm{HK} 2$ elevated aerobic glycolysis and facilitated proliferation by blocking apoptosis and especially cause resistance of NPC cells to radiation therapy, leading to the poor overall survival of NPC patients $^{7}$. On the other hand, EBV-LMP1 promote VEGF expression and angiogenesis through the JNKs/cJun signaling pathway, and LMP1-targeted DNAzyme increases the radiosensitivity of NPC by inhibiting HIF1/VEGF activity. These studies analyzed the molecular mechanism of EBV-LMP1 involved in radioresistance of NPC cells from the DNA damage repair, metabolism, and angiogenesis ${ }^{8}$. In present study, we further explored the protective autophagy mediated by LMP1 through ERK/HIF1 $\alpha /$ BNIP3 pathway to promote the radioresistance of tumor cells. These studies fully show that LMP1 can play an important role in NPC radioresistance through a variety of mechanisms, and our current work expands the understanding of the molecular mechanism of LMP1 in the NPC development.

\section{Materials and methods}

\section{Cell cultures, transfection and reagents}

CNE1, HNE2 and HK1 are LMP1 negative NPC cell lines. CNE1-LMP1(CM), HNE2-LMP1(HM) and HK1LMP1(KM) are cell lines that are stably transfected with EBV-LMP1 $1^{6,7}$. The source of these cell line had been identified and authenticated, and the mycoplasma contamination has been tested. The cells were cultured in RPMI-1640 medium supplemented with $10 \%$ fetal bovine serum (FBS, Gibco BRL, Gaithersburg, MD, USA), $100 \mathrm{IU} / \mathrm{mL}$ penicillin, $100 \mathrm{mg} / \mathrm{mL}$ streptomycin, and $2 \mathrm{mM} / \mathrm{L}$ L-glutamine in a humidified atmosphere of $5 \% \mathrm{CO}_{2}$ at $37{ }^{\circ} \mathrm{C}$. The cell transfection used Lipofectamine 2000 (Invitrogen, Carlsbad, CA, USA) according to the manufacturer's instructions. The pGV141-LMP1-wt were purchased from Genechem (Shanghai, China). The shRNA targeting BNIP3 and control-shRNA were synthesized by Ribo BioTechnology (Guangzhou, China), and the sequences of shRNA are described in Table S1. The AKT inhibitor (LY294002), JAK3 inhibitor (WHIP131), p38 inhibitor (SB203580), MEK inhibitor (PD98059), and JNKs inhibitor (SP600125) were purchased from Selleck Chemicals (Houston, TX, USA). The HIF1 $\alpha$ inhibitor (2ME2) was obtained from Cayman Chemical (Ann Arbor, MI, USA), and MitoTracker ${ }^{\mathrm{TM}}$ Red was purchased from Invitrogen (M7512).

\section{Transmission electron microscopy}

The NPC cells were harvested by trypsin digestion and fixed with $2.5 \%$ glutaraldehyde on ice for $2 \mathrm{~h}$, followed by postfixation in $2 \%$ osmiumtetroxide. Then, the cells were immersed in SPIPON812 resin after dehydrating with sequential washes in $50,70,90,95$, and $100 \%$ acetone. The ultrathin sections (50-100 nm) were collected on copper grids and counterstained using 3\% uranyl acetate and leadcitrate. Images were taken with a Tecnai G2 Spirit TEM (FEI, Thermo Fisher Scientific, Waltham, MA, USA).

\section{Immunofluorescence}

The cells were transfected with pEGFP-LC3 plasmid (Addgene, plasmid\#: 24920). For fluorescence analysis, cell samples were visualized with confocal microscopy (LSM 510 META, Carl Zeiss, Germany). The average value is the number of puncta in at least 50 randomly selected individual cells under each condition.

\section{Quantitative RT-PCR}

Total RNA was isolated from cells with the TRIzol reagent (15596026, Invitrogen) and reverse transcribed into cDNA (K1621, Thermo Fisher Scientific), according to the manufacturer's instructions. Quantitative PCR was performed using an ABI 7500 instrument (Foster City, CA, USA) with TaqManTM Gene Expression Master Mix (4369016, Thermo Fisher Scientific). For the qPCR array, the selected 88 autophage-associated genes were mainly referred to the gene information from the commercial chip such as PAHS-084Z autophagy gene chip (SABioscience, Frederick, MD, USA), and autophageassociated genes reported in the literatures. $\beta$-Actin was used as an internal control. The primers are listed in Table S2.

\section{Western blotting}

Cells were lysed with IP lysis buffer containing a 10\% cocktail (B14001, Bimake, Houston, TX, USA), and the protein concentration was determined using a BCA kit (Pierce Chemical, Rockford, IL, USA). SDS-PAGE was performed using $30-50 \mu \mathrm{g}$ of total cell protein. Then, the protein was transferred to a PVDF membrane, blocked with $5 \%$ skim milk at room temperature, and incubated with the indicated antibodies. The primary antibodies used were anti-LMP1 (M0897, 1:500) purchased from DAKO (Glostrup, Denmark), and anti-LC3 obtained from NOVUS (NB100-2220, 1:500, Littleton, CO, USA). AntiBNIP3 (ab10433, 1:1000) and anti-Beclin1 (ab207612, 1:1000) were purchased from Abcam (Cambridge, MA, USA). Anti-p-Akt (ser473, 9270, 1:1000), anti-p-ERK1/2 (4370, 1:1000), anti-p-JNK (Thr183/Tyr185, 9251, 1:1000), anti-p-JAK3 (Tyr980/981, 5031, 1:1000), antirabbit IgG-HRP (14708, 1:2000), and anti-mouse 
IgG-HRP (14709, 1:2000) antibodies were obtained from cell signaling technology (Danvers, MA, USA). Anti-Bcl-2 (sc-7382, 1:500), anti-p-P38 (thr180/try182, sc-4511, 1:1000), anti-HIF1 $\alpha$ (sc-53546, 1:500), and anti- $\beta$-actin (sc-8432, 1:2000) antibodies were obtained from Santa Cruz Biotechnology (Dallas, Texas, USA). Blots were analyzed using a chemiluminescence imaging system (Bio-Rad, Hercules, CA, USA).

\section{Co-immunoprecipitation analysis (Co-IP)}

For the Co-IP, cell lysates were clarified by immunomagnetic separation and incubated with the indicated antibody plus Dynabeads ${ }^{\circledR}$ Protein A (Thermo Scientific, 10002D) at $4{ }^{\circ} \mathrm{C}$ overnight to form immunocomplexes. After extensive washing with lysis buffer, the immunocomplexes were analyzed by western blotting, as described above.

\section{Cell viability assay}

Cell viability was measured by MTS kit (G5421, Promega, Madison, WI, USA) following the manufacturer's instruction. Cells were cultured in a 96-well plate with differently treatments for the indicated time, and then the assay solution was added for an incubation of $1.5 \mathrm{~h}$. The samples were measured by a Microplate Reader (BioTek ELx800, Winooski, VT, USA) at $490 \mathrm{~nm}$.

\section{Flow cytometry analysis}

$1-5 \times 10^{5}$ cells from each sample were digested with $0.25 \%$ trypsin and fixed in $70 \%$ cold ethanol overnight after resuspending in D-hanks three times. The cells were subsequently collected and were treated according to the instructions of the Annexin-V FITC/PI Apoptosis Detection Kit (KGA107, KeyGEN BioTECH, Jiangsu, China). All samples were analyzed using flow cytometry (MoFlo XDP, Beckman Coulter, Miami, FL, USA) according to the manufacturer's protocols.

\section{X-ray irradiation}

The cells and mice irradiation experiments were carried out on a PXI X-RAD 225 system (Precision X-ray Inc., North Branford, CT) at indicated dosages.

\section{Colony formation assay}

The radiosensitivity was measured by colony-formation assay as previously described ${ }^{36}$. Briefly, a gradient number of NPC cells with different treatments were seeded into 6well dishes and cultured for another $24 \mathrm{~h}$. After the cells were attached to the plates, the cells were irradiated with $\mathrm{X}$-rays $(0,2,4$, or $6 \mathrm{~Gy})$, then were further cultured for 2 weeks to allow colony formation. Colonies in dishes were stained with $0.0125 \%$ crystal violet (Sigma-Aldrich, St. Louis, MO, USA), and counted for the number of surviving colonies (defined as a colony with $>50$ cells).
The data were analyzed using the linear-quadratic model, and the surviving fraction was calculated as the ratio of the plating efficiency of the treated cells compared to control cells using GraphPad Prism (GraphPad Software, La Jolla, CA, USA).

\section{Animal experiments}

Animal experiments were conducted with the approval of the Institutional Animal Care and Use Committee of the Xiangya School of Medicine of Central South University and conform to the legal mandates and federal guidelines for the care and maintenance of laboratory animals. Here, no blinding was done in animal experiments. Twelve six-week-old female athymic nude mice $(\mathrm{BALB} / \mathrm{C})$ were injected with $5 \times 10^{6} \mathrm{CM}$ cells or $5 \times 10^{6}$ CM-shBNIP3 cells resuspended in $100 \mu \mathrm{L}$ PBS. Tumor volumes were calculated using the formula: (length $*$ width $\left.^{2}\right) *(\pi / 6)$. The animals were randomly divided into control group and the irradiation treatment (IR) group $(n=3$ per group) when the tumor volume reached $60-100 \mathrm{~mm}^{3}$, and $6 \mathrm{~Gy}$ local irradiation was administered in groups of mice subjected to IR. After 3 weeks, the mice were killed, and the tumors were collected and fixed with $10 \%$ buffered formalin for IHC analysis.

\section{Immunohistochemistry (IHC)}

A total of 27 Paraffin-embedded tumor tissue samples with clinical details of NPC patients (from 2017 to 2019) were collected from the Pathology Department of Xiangya Hospital (Table S3). IHC was performed using a Histomouse SP Broad Spectrum DAB kit (Invitrogen-Zymed, Carlsbad, CA, USA) following the manufacturer's instructions. The stained sections were independently examined by two of the authors (Z.Z. and J.P.). A semiquantitative evaluation of the positivity of each protein by IHC was performed using a method described previously ${ }^{8}$.

\section{Statistical analysis}

Statistical analysis was performed with the SPSS statistical software program (ver.19.0). Data are presented as means \pm SD. Differences between various groups were evaluated using a two-tailed Student's $t$-test and a $p$ value $<0.05$ was considered statistically significant.

\footnotetext{
Author details

${ }^{1}$ Department of Oncology, Key Laboratory of Carcinogenesis and Cancer Invasion of Ministry of Education, Xiangya Hospital, Central South University, Changsha, China. ${ }^{2}$ Cancer Research Institute, School of Basic Medicine Science, Central South University, Changsha, China. ${ }^{3}$ Department of Dermatology, Xiangya Hospital, Central South University, Changsha, China. ${ }^{4}$ Department of Pathology, Xiangya Hospital, Central South University, Changsha, China. ${ }^{5}$ Institute of Molecular Medicine and Oncology, College of Biology, Hunan University, Changsha, China. ${ }^{6}$ Hunan Key Laboratory of Oncotarget Gene, Hunan Cancer Hospital and The Affiliated Cancer Hospital of Xiangya School of Medicine, Central South University, Changsha, China
} 


\section{Author contributions}

L.Y. performed study concept and design; S.X., Z.Z., X.P., X.T., P.Z. and K.Z. performed development of methodology and writing, review, and revision of the paper; S.X., Z.Z. and J.P. provided acquisition, analysis and interpretation of data, and statistical analysis; D.L. and L.S. provided technical and material support. All authors read and approved the final paper.

\section{Funding}

This study was supported by grants from the National Natural Science Foundation of China (No. 81372182, 81672761) and the Natural Science Foundation of Hunan Province (2018JJ2545).

\section{Conflict of interest}

The authors declare no competing interests.

\section{Ethics statement}

Animal experiments were conducted with the approval of the Institutional Animal Care and Use Committee of the Xiangya School of Medicine of Central South University and conform to the legal mandates and federal guidelines for the care and maintenance of laboratory animals.

\section{Publisher's note}

Springer Nature remains neutral with regard to jurisdictional claims in published maps and institutional affiliations.

Supplementary information The online version contains supplementary material available at https://doi.org/10.1038/s41419-021-03639-2.

Received: 18 November 2020 Revised: 14 March 2021 Accepted: 18 March 2021

Published online: 01 April 2021

\section{References}

1. Chen, Y. P. et al. Nasopharyngeal carcinoma. Lancet 394, 64-80 (2019).

2. Lee, H. M., Okuda, K. S., Gonzalez, F. E. \& Patel, V. Current perspectives on nasopharyngeal carcinoma. Adv. Exp. Med. Biol. 1164, 11-34 (2019).

3. Young, L. S., Yap, L. F. \& Murray, P. G. Epstein-Barr virus: more than 50 years old and still providing surprises. Nat. Rev. Cancer 16, 789-802 (2016).

4. Wang, L. W., Jiang, S. \& Gewurz, B. E. Epstein-Barr virus LMP1-mediated oncogenicity. J. Virol. 91, 21 (2017).

5. Tao, Y. et al. Novel roles and therapeutic targets of Epstein-Barr virus-encoded latent membrane protein 1-induced oncogenesis in nasopharyngeal carcinoma. Expert Rev. Mol. Med. 17, e15 (2015).

6. Lu, J. et al. EBV-LMP1 suppresses the DNA damage response through DNAPK/AMPK signaling to promote radioresistance in nasopharyngeal carcinoma. Cancer Lett. 380, 191-200 (2016).

7. Xiao, L. et al. Targeting Epstein-Barr virus oncoprotein LMP1-mediated glycolysis sensitizes nasopharyngeal carcinoma to radiation therapy. Oncogene 33, 4568-4578 (2014)

8. Yang, L. et al. EBV-LMP1 targeted DNAzyme enhances radiosensitivity by inhibiting tumor angiogenesis via the JNKs/HIF-1 pathway in nasopharyngeal carcinoma. Oncotarget 6, 5804-5817 (2015).

9. Guo, J. Y., Xia, B. \& White, E. Autophagy-mediated tumor promotion. Cell 155 1216-1219 (2013).

10. Hale, A. N., Ledbetter, D. J., Gawriluk, T. R. \& Rucker, E. B. Autophagy: regulation and role in development. Autophagy 9, 951-972 (2013).

11. Levy, J. M. M., Towers, C. G. \& Thorburn, A. Targeting autophagy in cancer. Nat. Rev. Cancer 17, 528-542 (2017).

12. Mulcahy Levy, J. M. \& Thorburn, A. Autophagy in cancer: moving from understanding mechanism to improving therapy responses in patients. Cell Death Differ. 27, 843-857 (2020).
13. Wang, F. et al. SMAD4 gene mutation renders pancreatic cancer resistance to radiotherapy through promotion of autophagy. Clin. Cancer Res. 24 3176-3185 (2018)

14. Chen, X. et al. Autophagy enhanced the radioresistance of non-small cell lung cancer by regulating ROS level under hypoxia condition. Int. J. Radiat. Biol. 93, 764-770 (2017)

15. Lee, D. Y. \& Sugden, B. The latent membrane protein 1 oncogene modifies B-cell physiology by regulating autophagy. Oncogene 27, 2833-2842 (2008).

16. Vasagiri, N. \& Kutala, V. K. Structure, function, and epigenetic regulation of BNIP3: a pathophysiological relevance. Mol. Biol. Rep. 41, 7705-7714 (2014).

17. Zhan, Y. \& Fan, S. Multiple mechanisms involving in radioresistance of nasopharyngeal carcinoma. J. Cancer 11, 4193-4204 (2020).

18. Yang, L. et al. Sevoflurane postconditioning alleviates hypoxia-reoxygenation injury of cardiomyocytes by promoting mitochondrial autophagy through the HIF-1/BNIP3 signaling pathway. PeerJ 7, e7165 (2019).

19. Sun, L. et al. Upregulation of BNIP3 mediated by ERK/HIF-1alpha pathway induces autophagy and contributes to anoikis resistance of hepatocellular carcinoma cells. Future Oncol. 10, 1387-1398 (2014).

20. Bellot, G. et al. Hypoxia-induced autophagy is mediated through hypoxiainducible factor induction of BNIP3 and BNIP3L via their BH3 domains. Mol. Cell Biol. 29, 2570-2581 (2009).

21. Tracy, K. \& Macleod, K. F. Regulation of mitochondrial integrity, autophagy and cell survival by BNIP3. Autophagy 3, 616-619 (2007).

22. Kuwahara, Y. et al. Enhancement of autophagy is a potential modality for tumors refractory to radiotherapy. Cell Death Dis. 2, e177 (2011).

23. Zhuang, W. et al. Induction of autophagy promotes differentiation of gliomainitiating cells and their radiosensitivity. Int J. Cancer 129, 2720-2731 (2011).

24. Chaachouay, $\mathrm{H}$. et al. Autophagy contributes to resistance of tumor cells to ionizing radiation. Radiother. Oncol. 99, 287-292 (2011).

25. Ko, A. et al. Autophagy inhibition radiosensitizes in vitro, yet reduces radioresponses in vivo due to deficient immunogenic signalling. Cell Death Differ. 21, 92-99 (2014).

26. Liu, F. et al. Verticillin A overcomes apoptosis resistance in human colon carcinoma through DNA methylation-dependent upregulation of BNIP3. Cancer Res. 71, 6807-6816 (2011).

27. Du, $Y$. et al. MicroRNA-145 induces apoptosis of glioma cells by targeting BNIP3 and Notch signaling. Oncotarget 8, 61510-61527 (2017).

28. Burton, T. R., Eisenstat, D. D. \& Gibson, S. B. BNIP3 (Bcl-2 19 kDa interacting protein) acts as transcriptional repressor of apoptosis-inducing factor expression preventing cell death in human malignant gliomas. J. Neurosci. 29, 4189-4199 (2009).

29. Chourasia, A. H. et al. Mitophagy defects arising from BNip3 loss promote mammary tumor progression to metastasis. EMBO Rep. 16, 1145-1163 (2015).

30. Wu, S. Y. et al. Ras-related tumorigenesis is suppressed by BNIP3-mediated autophagy through inhibition of cell proliferation. Neoplasia 13, 1171-1182 (2011).

31. Nollet, E. A. et al. Androgen receptor-induced integrin alpha6beta1 and Bnip3 promote survival and resistance to PI3K inhibitors in castration-resistant prostate cancer. Oncogene 39, 5390-5404 (2020).

32. Li, Q., Qi, F., Meng, X., Zhu, C. \& Gao, Y. Mst1 regulates colorectal cancer stress response via inhibiting Bnip3-related mitophagy by activation of JNK/p53 pathway. Cell Biol. Toxicol. 34, 263-277 (2018).

33. Riis, S., Murray, J. B. \& O'Connor, R. IGF-1 signalling regulates mitochondria dynamics and turnover through a conserved GSK-3beta-Nrf2-BNIP3 pathway. Cells 9, 147 (2020)

34. Zhu, Y. et al. Modulation of serines 17 and 24 in the LC3-interacting region of Bnip3 determines pro-sunvival mitophagy versus apoptosis. J. Biol. Chem. 288, 1099-1113 (2013).

35. Shi, R. Y. et al. BNIP3 interacting with LC3 triggers excessive mitophagy in delayed neuronal death in stroke. CNS Neurosci. Ther. 20, 1045-1055 (2014).

36. $\mathrm{Ma}, \mathrm{X}$. et al. Down-regulation of EBV-LMP1 radio-sensitizes nasal pharyngeal carcinoma cells via NF-kappaB regulated ATM expression. PLoS ONE 6, e24647 (2011). 